\title{
Refractive Laser Triangulation and Photometric Stereo in Underwater Environment
}

\author{
Hao Fan, Lin Qi, Yakun Ju, Junyu Dong*, Hui Yu
}

\begin{abstract}
Laser triangulation and photometric stereo are popular optical 3D reconstruction methods but bear limitations in underwater environment because of the refraction phenomenon. Refraction bends the usually straight rays of light to another directions in the interface of a flat underwater housing. It causes the camera to capture the virtual object points instead of the real ones, so that the commonly used pinhole camera model is invalid. Therefore, in this paper, we introduce a flat refractive model for describing the geometric relation accurately between the virtual object points and the real ones, which can correct the distortions in underwater $3 D$ reconstruction methods. The parameters of model can be estimated in a calibration step with a standard chessboard. Then the proposed geometric relation is used for rebuilding underwater three-dimensional relationship in laser triangulation and photometric stereo. The experimental results indicate the effectiveness of our methods in underwater 3D reconstruction.
\end{abstract}

Index Terms-refractive model, laser triangulation, photometric stereo.

\section{INTRODUCTION}

$\mathbf{M}$ ANY underwater tasks, such as seabed mapping, archaeological surveys, and oil pipelines inspection, require high-precision 3D information of the target object, where optical methods provide better solution than acoustic ones. Laser triangulation and photometric stereo are classical optical techniques for high-precision 3D reconstruction, which have been successfully applied in the air medium. However, employing them in underwater environment confronts problems such as image degrading and the refraction occurring at the interface of different mediums. These problems, especially the refraction, cause erroneous estimation of surface shape in 3D reconstruction results.

In underwater environment, cameras are usually protected in a watertight housing. Light rays reflected by the object in the water are refracted when it goes through the flat housing glass in front of the camera [1]. The classical pinhole camera model (also called single viewpoint (SVP) model) cannot hold true because of the refraction. Thus, modeling the light propagation in multimedia which extends these typical 3D reconstruction techniques to underwater environment needs to be further investigated.

Laser triangulation uses the triangulation relationship between the structured light and the camera to solve for the scene geometry. Refraction results in systematic errors (deformed shapes) in underwater laser triangulation. Previous literatures [2], [3] mostly regarded the underwater imaging

Ocean University of China, College of Information Science and Engineering, 238 Songling Road, Qingdao, China, 266100.

*Corresponding author: Junyu Dong(dongjunyu@ouc.edu.cn). system as the perspective projection, and sought suitable corrections to the refractive effect. In some works [4], [5], the refractive effect was absorbed by the intrinsic camera parameters - the focal length and lens distortions. However, it is impossible to remove the refraction error completely in conventional camera calibration methods [6]. Recently, some researches [1], [7] proposed geometrically driven approaches, which studied the light propagation in multimedia. These geometric-based approaches are more accurate than these approximating perspective projection. Nevertheless, few works focused on improving underwater laser triangulation by using a suitable physical-based refractive model.

Photometric stereo estimates surface normal and height by using more than three images taken from the same viewpoint but different illumination directions [8], [9]. Researchers have employed light propagation model to handling the effects caused by light attenuation and scattering [10], [11], [12]. $3 \mathrm{D}$ reconstruction in pixel-level can be completed in a turbid medium, but real locations of the captured object can not be obtained without a suitable refractive model. However, few works considered the refractive effect in underwater photometric stereo.

In this paper, we employ a flat refractive model to accommodate the refractive effect in underwater 3D reconstruction. According to the SVP model, the captured image reflects the virtual object points instead of the real ones. Thus, we assume the virtual object point is right above the real one, and establish a "virtual-real" relationship between them. There are two parameters in the model: the camera-glass distance and the refractive index of the water medium. These parameters are then estimated in a calibration step. Based on the model above, we rebuild the triangulation relationship in underwater laser triangulation, and estimate actual three-dimensional locations of the captured object using underwater photometric stereo.

The main contribution of this paper can be summarized as the following three aspects. First, we introduce a flat refractive model and establish a "virtual-real" relationship, which is more suitable to solve the refractive effect in underwater 3D reconstruction. Second, we propose an underwater laser triangulation approach based on the "virtual-real" relationship to improve the reconstruction accuracy. Third, according to the "virtual-real" relationship, we present an algorithm to estimate the actual three-dimensional locations using underwater photometric stereo. Experiments show the high-level precision of underwater 3D reconstruction can be achieved by the proposed refraction-based laser triangulation and photometric stereo.

This paper is organized into seven sections. Section 2 reviews the related work and gives a short view of the SVP 
model. Section 3 presents the flat refractive model and the "virtual-real" relationship. Section 4 gives our calibration method for model parameter estimation. Section 5 proposes the underwater laser triangulation and photometric stereo methods. Then Section 6 discusses the performance of the proposed methods with other related methods. Finally, Section 7 makes concluding remarks on the proposed methods.

\section{BACKGROUND}

\section{A. Related Work}

Underwater laser triangulation: Laser triangulation is used for obtaining accurate three-dimensional information. Laser line scan system (LLS) [13], [14] based on laser triangulation is one of the most popular approaches for underwater 3D mapping [4], [15]. Previous approaches [2], [5], [16] attempted to calibrate the laser mapping relationship with some known projected points. But these approaches can not remove the effective effect completely, because the refraction caused deformation is non-linear and hard to be fitted. In [10], the light ray of each image pixel was calibrated in underwater environment, then the three-dimensional position could be accurately estimated combining with the laser plane equation. But the calibration step was cumbersome. Recently, Chi et al. [7] relaxed the underwater image to the air based on a refractive model, by which they easily calibrated the structured light system in air. But the effectiveness of the refractive model relied on an approximate assumption, which made the computation easy but brought tiny deviations to the final results. In this work, we rebuild the triangulation based on a geometric relation without any approximations to obtain the three-dimensional information of high accuracy.

Underwater photometric stereo: Photometric stereo can estimate the surface shape with more details. However, considering the underwater scenes with complex elements such as light attenuation, scattering, refraction and so on, photometric stereo cannot be easily work. In [9], Kolagani et al. firstly applied photometric stereo in underwater robots. However, their work did not consider backscatter, and the optimization method was not suitable in an underwater environment because of the refraction. Recently, researchers established more accurate light model to reduce the effect caused by light attenuation and scattering [10], [11], [12]. However, few works considered the refractive effect. In this work, we achieved accurate three-dimensional locations from the de-scattered photometric-stereo results based on a refractive model.

Underwater refractive model: In underwater environment, the SVP model does not work due to light refraction. There are two kinds of approaches to solve the problem [5]: one is based on seeking corrections to compensate for the refractive effects, and the other is on the calculation of geometric refractive models. The first approach was employed in most works [4], [17]. They represented the refractive effect as the camera parameters - the focal length and the lens distortions. However, there is difference between the refraction and the lens distortion[6]. The geometric methods are more applicable for accurate 3D estimation. Different flat refractive models [3], [5], [18] were proposed to describe the light propagation in underwater scenes. In [3], Treibitz et al. presented a physicalbased model, which used an SVP approximation for a flatinterface system. In [5], Telem and Filin proposed a refractive model to describe the geometry distortions and estimate the additional parameters without any approximation or reduction. Moreover, researchers have successfully applied refractive models in underwater stereo vision[19], [20] and Structure from Motion (SFM)[21].

In this paper, we also introduce a physical-based model to solve the effect of refraction in underwater laser triangulation and photometric stereo. Compared to the prior models, especially the refractive model in [1], we describe the threedimensional geometric relations between the virtual object points and the real ones instead of the perspective center or the focal length. And the proposed model is more suitable for underwater laser triangulation and photometric stereo.

\section{B. The SVP Model}

The camera behind the flat interface obeys the perspective projection. For a short review about the imaging model [22], there are four coordinate systems in the transformation from the coordinate of a scene point to its physical image coordinate. With the extrinsic parameters, the world coordinate system is related to the camera coordinate system. With the intrinsic parameters, the camera coordinate system and the normalized image coordinate system are related to the physical image coordinate system. The change in coordinates between the physical image frame and the normalized one is defined as:

$$
p=K \hat{p} .
$$

Here, the physical image coordinate vector is $\hat{p}=[u, v, 1]^{T}$, where $T$ denotes the transposition. The normalized image coordinate vector is $p=[x, y, 1]^{T}$. The matrix $K$ denotes the intrinsic parameters of the camera:

$$
K=\left[\begin{array}{ccc}
\alpha & -\alpha \cot \theta & u_{0} \\
0 & \beta / \sin \theta & v_{0} \\
0 & 0 & 1
\end{array}\right]
$$

where $u_{0}$ and $v_{0}$ define the position (in pixel units) of the principal point; $\alpha$ and $\beta$ are related to the focal length of $x$ and $y$-axes in pixel units; and $\theta$ is the angle between the two image axes [22]. Thus, if the coordinate of a scene point in the camera coordinate system is defined as $P=[x, y, z, 1]^{T}$, we obtain:

$$
p=\frac{1}{z} M P, \quad \text { where } \quad M=\left(\begin{array}{ll}
K & 0
\end{array}\right) .
$$

Furthermore, we can transform a scene point from the the world coordinate system to a physical image coordinate in the physical image coordinate system with the extrinsic parameters:

$$
p=\frac{1}{z} M P, \quad \text { where } \quad M=K\left(\begin{array}{ll}
R & t
\end{array}\right),
$$

where $R$ is a rotation matrix, $t$ is a translation vector, and $P$ denotes the coordinate vector in the world coordinate system. Both of the intrinsic and extrinsic parameters are calibrated using Zhang's method [23] in this work. 


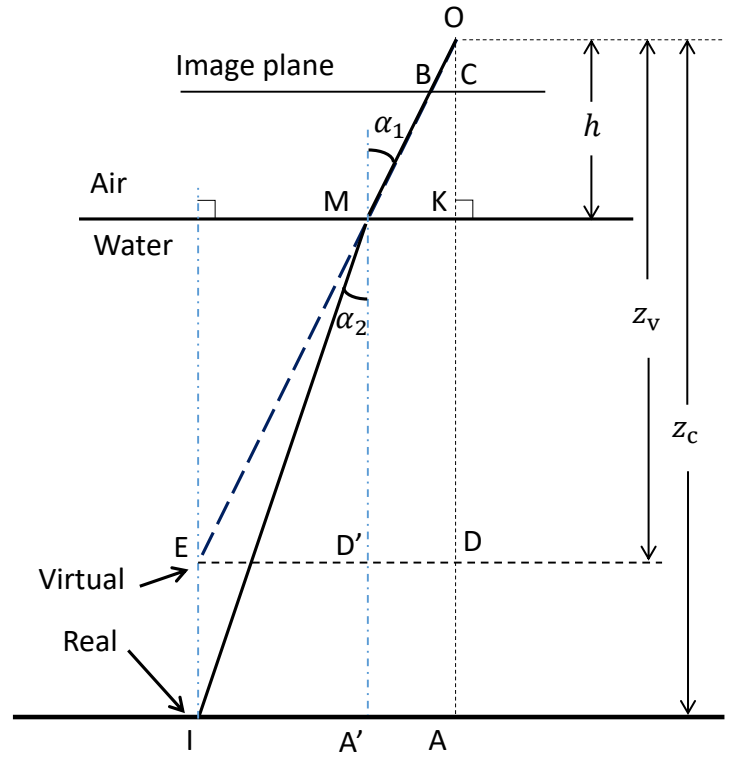

Fig. 1. The physical-based refractive model.

\section{PhysiCAL-BASED REFRACTIVE MODEL}

In case of the flat protecting housing device, light rays are refracted in the housing interface in underwater environment. Since the refraction is non-linear, the common Direct Linear Transformation(DLT) method[6] cannot perform an accurate calibration. To solve the problem, we introduce a physicalbased refractive model, that fits with the research in [1]. For better applied in underwater 3D reconstruction, we give a new explanation for the refraction in the air-water interface, and propose a "virtual-real" relationship in the model.

The geometric relation of the refractive model is illustrated in Fig. 1. The camera is protected in a watertight housing. The camera's optical axis is perpendicular to the housing interface. According to the perspective projection, the virtual point can be set in any position along the extension line of $O M$. To simplify the calculation, we assume the virtual point is right above the real one, and the link line of the two points is perpendicular to housing interface. The model is characterized by following parameters: $h$, the distance between the perspective center $O$ and the housing interface; $\alpha_{1}$, the angle of incidence; $\alpha_{2}$, the angle of refraction in water; $I$, the real object point $\left(x_{c}, y_{c}, z_{c}\right)$ (in the camera coordinate system); and $E$, the virtual object point $\left(x_{v}, y_{v}, z_{v}\right)$ related to the point $I$. In this model, there obviously exits relations: $x_{c}=x_{v}, y_{c}=y_{v}$. And our objective is to build the "virtualreal" relation between $z_{v}$ and $z_{c}$.

Assuming that the optical axis, the incident ray, and the refracted ray are all on the same plane. And following Snell's law of refraction, the refractive index $n$ can be written as:

$$
n=\frac{\sin \alpha_{1}}{\sin \alpha_{2}}
$$

According to the geometric relation, the angular quantities can be expressed as:

$$
\begin{aligned}
\sin \alpha_{1} & =\frac{E D^{\prime}}{M E}, \\
\sin \alpha_{2} & =\frac{I A^{\prime}}{M I} .
\end{aligned}
$$

In Fig. 1, there exit geometric relations: $E D=I A, E D^{\prime}=$ $I A^{\prime}, M D^{\prime}=K D, M A^{\prime}=K A$, and $A A^{\prime}=D D^{\prime}=M K$. Thus, from Eqs. (5) and (6), the refractive index can be written as:

$$
n=\frac{E D^{\prime} \times M I}{I A^{\prime} \times M E}=\frac{M I}{M E} .
$$

Since $z_{v}=O D$ and $z_{c}=O A$, the "virtual-real" relation can be estimated from the geometric relations of $O A$ and $O D$. Obviously, there is a common factor $O K$ in $O A$ and $O D$ :

$$
\begin{aligned}
& O A=O K+K A, \\
& O D=O K+K D,
\end{aligned}
$$

where $O K$ denotes the camera-glass distance $h$. Next, we will find the relationship between $K A$ and $K D$. Following the geometric relation, $K A$ can be expressed as:

$$
\begin{aligned}
K A & =\sqrt{M I^{2}-I A^{\prime 2}} \\
& =\sqrt{n^{2} \times M E^{2}-I A^{\prime 2}} \\
& =\sqrt{n^{2} \times\left(M D^{\prime 2}+E D^{\prime 2}\right)-I A^{\prime 2}} \\
& =\sqrt{n^{2} \times\left(K D^{2}+I A^{\prime 2}\right)-I A^{\prime 2}} \\
& =\sqrt{n^{2} \times K D^{2}+\left(n^{2}-1\right) \times I A^{\prime 2}},
\end{aligned}
$$

where $K D=O D-O K$ and $I A^{\prime}=I A-A A^{\prime}=I A-M K$. Then, $K A$ can be redefined as:

$$
K A=\sqrt{n^{2} \times(O D-O K)^{2}+\left(n^{2}-1\right) \times(I A-M K)^{2}} .
$$

From Eqs. (8) and (10), $O A$ can be expressed as:

$O A=O K+\sqrt{n^{2} \times(O D-O K)^{2}+\left(n^{2}-1\right) \times(I A-M K)^{2}}$.

Thus, the relation between $z_{v}$ and $z_{c}$ can be estimated as:

$$
z_{c}=h+\sqrt{n^{2} \times\left(z_{v}-h\right)^{2}+\left(n^{2}-1\right) \times(I A-M K)^{2}} .
$$

Based on the perspective camera model, both points $I$ and $E$ are related to an image point $B\left(x_{u}, y_{u}\right)$ (in the normalized image coordinate system). The relations in the real point $I$, the virtual point $E$ and the related image point $B$ can be described as:

$$
\frac{B C}{O C}=\frac{M K}{O K}=\frac{E D}{O D},
$$

where $B C$ denotes the distance from the point $B$ to the image plane center $C$, and $O C=1$ in the normalized image coordinate system. From this equation, $M K$ can be expressed as

$$
M K=\frac{B C \times O K}{O C}=\sqrt{x_{u}^{2}+y_{u}^{2}} \times h .
$$

And $I A$ denotes the distance from point $I$ to the $X Y$ plane center $A$, that can be expressed as:

$$
I A=E D=\frac{B C \times O D}{O C}=\sqrt{x_{u}^{2}+y_{u}^{2}} \times z_{v} .
$$




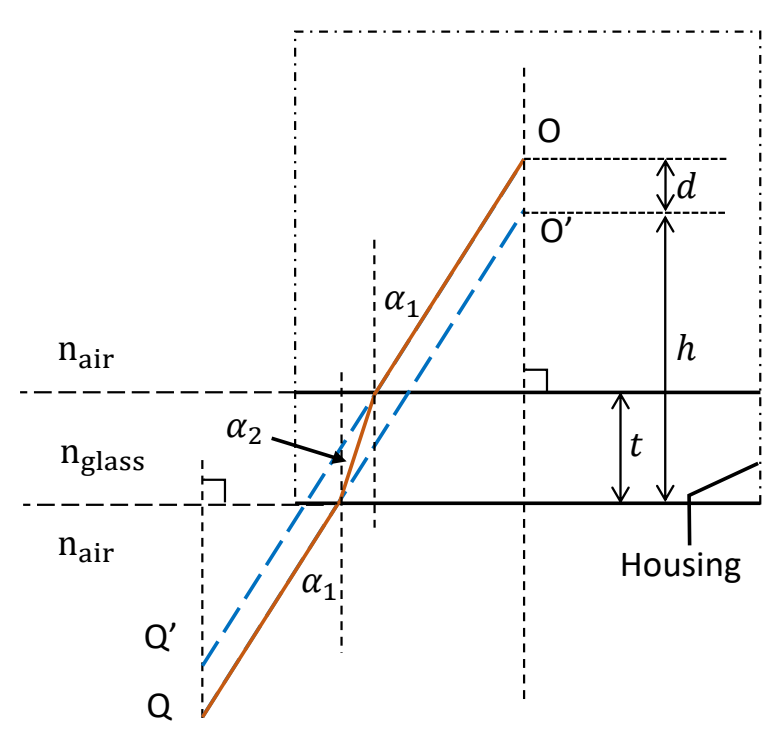

Fig. 2. Refraction in housing interface.

Finally, combining Eqs. (14), (15) with Eq. (12), the "virtual-real" relationship in this refractive model can be expressed as:

$$
\left\{\begin{array}{l}
x_{c}=x_{v} \\
y_{c}=y_{v} \\
z_{c}=h+\left(z_{v}-h\right) \times \sqrt{n^{2}+\left(n^{2}-1\right) \times\left(x_{u}^{2}+y_{u}^{2}\right)},
\end{array}\right.
$$

where $x_{v}=z_{v} \times x_{u}, y_{v}=z_{v} \times y_{u}$.

\section{A. Interface Thickness}

In the presented refractive model, we consider the camera and the housing device as a integral, and call it the housing camera. In addition to the deviation of the rays in the housing interface, the effect of the interface thickness on the imaging system is also studied.

As illustrated in Fig. 2, the optical axis of the camera is perpendicular to the housing interface. The light ray originated from object $Q$ is refracted through the glass toward the camera $O$. Point $Q^{\prime}$ is the virtual point right above point $Q$, and the link line of the two points is perpendicular to the housing interface. The ray line $O Q^{\prime}$ parallels with the ray line $O^{\prime} Q$. Without the glass, point $Q$ should be captured by camera $O^{\prime}$. In this housing model, $d$ denotes the distance between the perspective center $O$ and the virtual perspective center $O^{\prime}$; $h$ is virtual camera-glass distance, which denotes the distance from the virtual perspective center $O^{\prime}$ to the housing interface; $t$ is the actual interface thickness; $\alpha_{1}$ is the incidence angle in the air; and $\alpha_{2}$ is the angle between the normal vector to the interface and the ray in the glass. Thus, the distance $d$ can be expressed as:

$$
d=t \times\left(1-\frac{\tan \alpha_{2}}{\tan \alpha_{1}}\right) .
$$

According to Shell's law, $n_{\text {air }} \sin \alpha_{1}=n_{\text {glass }} \sin \alpha_{2}$. The distance $d$ can be redefined as a function depending on the

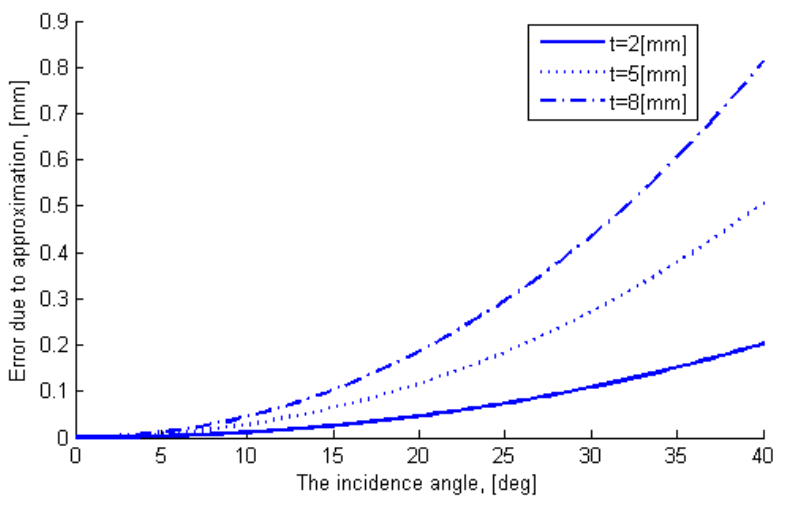

Fig. 3. Difference of $d$ is introduced from the refraction in housing interface with respect to an exact computation of the interface thickness ( $t$ ) effect.

incidence angle $\alpha_{1}$ and the interface thickness $t$ :

$$
d\left(\alpha_{1}\right)=t \times\left(1-\frac{\tan \left(\arcsin \left(\frac{n_{\text {air }}}{n_{\text {glas }}} \sin \alpha_{1}\right)\right)}{\tan \alpha_{1}}\right) .
$$

If all the virtual line $O^{\prime} Q$ are intersected at the same point $O^{\prime}$, we can take the point $O^{\prime}$ as the perspective center of the housing camera. However, the value of $d$ is varying due to the rate of $\tan \alpha_{2} / \tan \alpha_{1}$. And the difference depending on the incidence angle $\alpha_{1}$ can be expressed as:

$$
\triangle d\left(\alpha_{1}\right)=d\left(\alpha_{1}\right)-d(0) .
$$

Considering a glass housing with $n_{\text {glass }} \cong 1.5, n_{\text {air }} \cong 1$, setting $t=2,5,8 \mathrm{~mm}$, and restricting $\alpha_{1}<40^{\circ}$, Fig. 3 shows the difference function $\triangle d\left(\alpha_{1}\right)$. When $t=2 \mathrm{~mm}$, the difference is lower than $0.1 \mathrm{~mm}$ up to $28^{\circ}$. The value increases when the interface becomes thicker. For a $8 \mathrm{~mm}$ thickness, the maximum difference is lower than $0.2 \mathrm{~mm}$ up to $21^{\circ}$, and rise to $\sim 0.8 \mathrm{~mm}$ at the image boundaries. The difference is less than $1 \mathrm{~mm}$ when the thickness $d$ is smaller than $10 \mathrm{~mm}$. Compared to the virtual camera-glass distance $h$, this difference is so small that it can be negligible. So the effect of the interface thickness is neglected in our method.

\section{CALIBRATION OF MODEL PARAMETERS}

Calibration of the model parameters is conducted in two step. First, a "try" process estimates the intrinsic parameters of housing camera by using Zhang's method[23] with a regular calibration pattern - a black-and-white checkerboard. Then a "wet" process estimates additional parameters including the refractive index $n$ and the camera-glass distance $h$.

For estimating the underwater parameters $n$ and $h$, the calibration plate is placed under the housing camera with a fixed position, and is photographed in air and water respectively. Then two internal corners of the checkerboard pattern image are extracted as the key calibration points. In our method, the two selected points should be near to the optical axis, and have different distance along the optical axis ( $Z$ axis).

As shown in Fig. 4, two internal corners $P_{1}$ and $P_{2}$ are selected as the key calibration points. Depths $O A_{1}$ and $O A_{2}$ denote the real depth of the two points. Depths $O D_{1}$ and $O D_{2}$ denote the virtual depth related to the same points. 


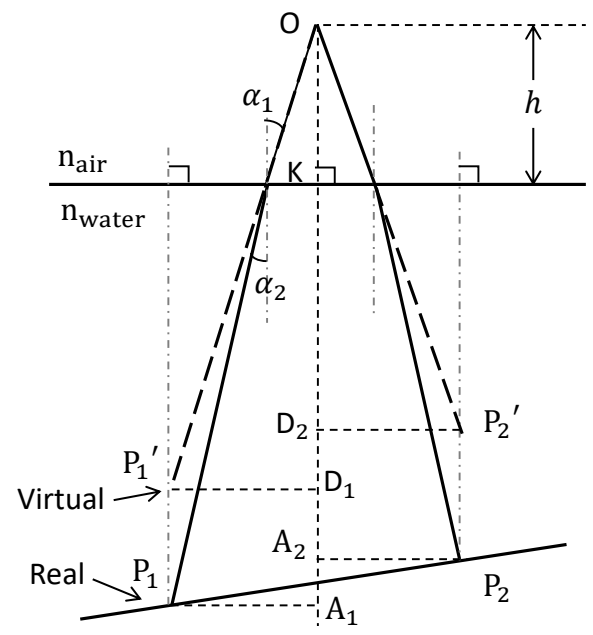

Fig. 4. Two-points calibration method for housing parameters - the refractive index $n$ and the camera-glass distance $h$.

The accurate values of these depths are all estimated using Zhang's method [23]. According to Shell's law, $n_{\text {air }} \sin \alpha_{1}=$ $n_{\text {water }} \sin \alpha_{2}$. Using the approximation $\tan \alpha \cong \sin \alpha$, which is applicable when $\alpha<8^{\circ}$, we can obtain:

$$
n=\frac{n_{\text {water }}}{n_{\text {air }}}=\frac{\sin \alpha_{1}}{\sin \alpha_{2}} \approx \frac{\tan \alpha_{1}}{\tan \alpha_{2}}=\frac{K A_{1}}{K D_{1}}=\frac{K A_{2}}{K D_{2}} .
$$

Based on the geometric relations (Fig. 4), we obtain:

$$
\begin{array}{ll}
K D_{1}=O D_{1}-O K \quad, \quad K A_{1}=O A_{1}-O K, \\
K D_{2}=O D_{2}-O K \quad, \quad K A_{2}=O A_{2}-O K,
\end{array}
$$

where $O K=h$.

Finally, the refractive index $n$ and the camera-glass distance $h$ can be estimated as:

$$
\begin{aligned}
h & =\frac{O A_{1} \times O D_{2}-O A_{2} \times O D_{1}}{O A_{1}+O D_{2}-O A_{2}-O D_{1}} ; \\
n & =\frac{O A_{1}-h}{O D_{1}-h} .
\end{aligned}
$$

\section{UNDERWATER LASER TRIANGULATION AND} Photometric Stereo Based on a Refractive Model

\section{A. Underwater Laser Triangulation}

Laser triangulation measures the reflected from a target surface to determine the position of the target. A laser device projects a spot of light to the target, and its reflection is focused via an optical lens on a light sensitive device. Triangulation in the laser line and the received ray is used to estimate the position of the target. However, the refraction breaks the triangulation. According to the "virtual-real" relationship in the refractive model, we rebuild the triangulation relations in underwater laser triangulation.

Fig. 5 shows the underwater laser triangulation system. The origin of the world coordinate system is away from the camera coordinate system along the principal optic axis with a distance $l$. The $X Y$ plane of the world coordinate system is parallel to the $X Y$ plane of the camera coordinate system. $X$ axises of the two systems are in the same direction. $Y$ and $Z$ axises are in the opposite direction.

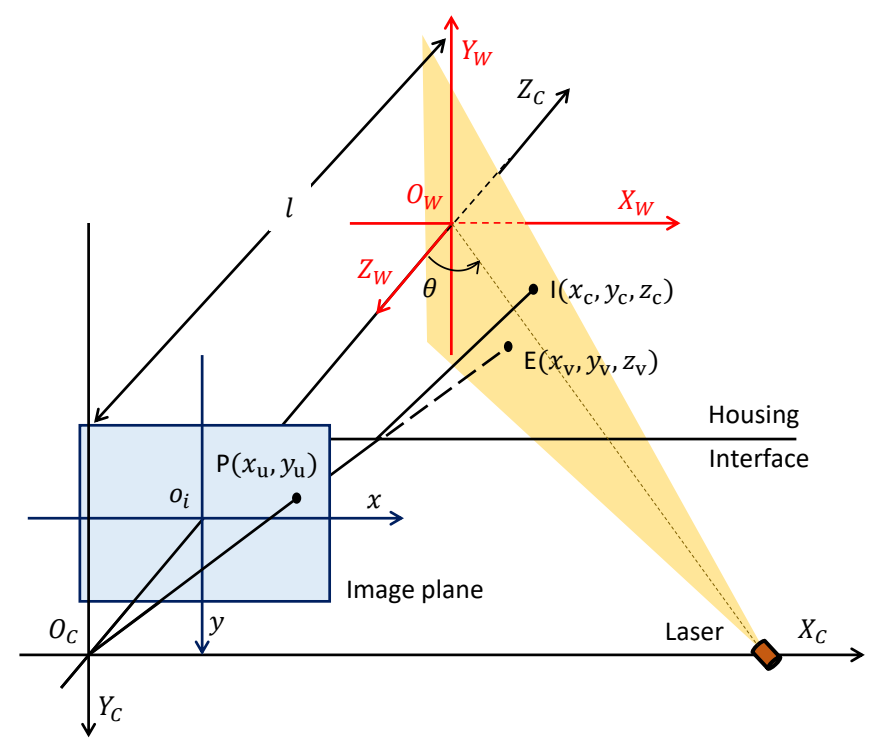

Fig. 5. Underwater laser triangulation in underwater environment.

The relation between the world coordinate system $\left(x_{w}, y_{w}, z_{w}\right)$ and the camera coordinate system $\left(x_{c}, y_{c}, z_{c}\right)$ is defined as:

$$
\left\{\begin{array}{l}
x_{w}=x_{c} \\
y_{w}=-y_{c} \\
z_{w}=l-z_{c} .
\end{array}\right.
$$

According to the SVP model, the captured reflected point, which is defined as $\left(x_{u}, y_{u}\right)$ in the normalized image coordinate system, is projected to a virtual position $\left(x_{v}, y_{v}, z_{v}\right)$ following the refractive model in the camera coordinate system. Thus, in the world coordinate system, the "virtual-real" relationship (Eq. (16)) can be written as:

$$
\left\{\begin{array}{ccc}
x_{w} & = & z_{v} \times x_{u} ; \\
y_{w} & = & -z_{v} \times y_{u} ; \\
z_{w} & = & l-h-\left(z_{v}-h\right) \times \delta,
\end{array}\right.
$$

where $\delta=\sqrt{n^{2}+\left(n^{2}-1\right) \times\left(x_{u}^{2}+y_{u}^{2}\right)}$.

For a simplified calculation, which does not influence the feasibility of the proposed algorithm, the laser plane is perpendicular to the housing interface, and travels through the Y-axis of the world coordinate system. The angle between the structured light plane and the principal optic axis is $\theta$. Thus, the laser plane in the world coordinate system can be defined as:

$$
x_{w}=z_{w} \tan \theta .
$$

Combining Eq. 26 and Eq. 27, we obtain the underwater laser triangulation relation:

$$
\left\{\begin{array}{l}
x_{w}=z_{v} \times x_{u} ; \\
y_{w}=-z_{v} \times y_{u} \\
z_{w}=z_{v} \times x_{u} / \tan \theta,
\end{array}\right.
$$

where $z_{v}=(l+h \times(\delta-1)) /\left(x_{u} / \tan \theta+\delta\right)$.

Finally, we can obtain the mapping relation from the physical image coordinate system to the world coordinate system for the reflected laser points using the calibrated intrinsic parameters, which are described in Sect. II-B. 


\section{B. Underwater Photometric Stereo}

Photometric stereo can recover object shapes in detail, and has been successfully applied in murky media [11], [24]. Prior works have considered the backscatter effect, and achieved fine results of pixel size. Nevertheless, there are few works that research the refractive effect in the underwater scene, and transform the reconstruction result to real size. The common approaches cannot rectify the shape deformation caused by refraction. Therefore, we propose an approach to correct the refractive influence based on the proposed "virtual-real" relationship in the refractive model.

According to the method in [11], after subtracting the estimated backscatter, we can obtain the surface normal $n$ of each point $(x, y, z)$ in the camera coordinate system from the direct component:

$$
E_{k}(x, y)-B_{k}(x, y)=\rho(z(x, y)) \mathbf{n} \cdot \mathbf{l}_{k},
$$

where $k$ denotes the $k$ th light source, $E_{k}(x, y)$ is the measured intensity, $B_{k}(x, y)$ is the backscatter component, $\rho(z(x, y))$ is the albedo related to the light-object distance, and $\mathbf{l}_{k}$ is the light direction of the $k$ th light source. Then we can estimate the height of the captured object in pixel size from the normal n by using [25].

Since photometric stereo estimates the shape from the shading information, which is not influenced by the light refraction, the estimated height is relatively true. Combining with some known points, we can transform the height from pixel size to real size. And the known points can be easily obtained using our underwater laser triangulation approach. A laser point $P\left(x_{0}, y_{0}, z_{0}\right)$ in camera coordinate system, which is related to pixel point $\left(x_{p}, y_{p}\right)$ in normalized image coordinate system, is extracted to realize the transition:

$$
H_{c}=\left(z_{0}+\tau \times h_{p}\right)-\tau \times H,
$$

where $H_{c}$ denotes the height of captured object in the camera coordinate system, $\tau\left(\approx x_{0} / x_{p}\right)$ is the conversion factor, $h_{p}$ is the height of point $P$ estimated by photometric stereo in pixel size, and $H$ denotes the surface hight produced by photometric stereo in pixel size.

According to the proposed "virtual-real" relationship (Eq. (16)), we can obtain:

$$
\left\{\begin{array}{l}
x_{c}=\frac{\left(z_{c}+(\delta-1) \times h\right)}{\delta} \times x_{u} \\
y_{c}=\frac{\left(z_{c}+(\delta-1) \times h\right)}{\delta} \times y_{u}
\end{array}\right.
$$

Now, combining with the real height $H_{c}$, the accurate 3D position of the captured object for each pixel can be estimated as:

$$
\left\{\begin{array}{lll}
x_{c} & = & \frac{\left(H_{c}+(\delta-1) \times h\right)}{\delta} \times x_{u} \\
y_{c} & = & \frac{\left(H_{c}+(\delta-1) \times h\right)}{\delta} \times y_{u} \\
z_{c} & = & H_{c}
\end{array}\right.
$$

Moreover, the result can be transformed to the world coordinate system like the process of underwater laser triangulation.

\section{EXPERIMENTS}

In order to validate the proposed underwater laser triangulation and photometric stereo approach, we designed a testing system in a controlled environment. The system included a tank of $1000 \mathrm{~mm} \times 1000 \mathrm{~mm} \times 1000 \mathrm{~mm}$, a camera (IDS UI$358 \times$ CP-C) with a housing device, a underwater line-laser device, and six underwater light sources. The camera was placed at the center of the system, surrounded by six LED light sources. The interface thickness of the housing device was $8 \mathrm{~mm}$. The light sources with an adjustable lighting direction were placed around a rotating circular orbit. And the laser device was placed alongside the camera with a known angle along the camera optical axis.

The laser and the camera were fixed together for underwater laser triangulation. Then the result of laser triangulation was used to enhance the result of underwater photometric stereo, which shared the same camera with the laser system. The angle to the optical axis of each light source was set to be 45 degree. The distance from the object to the camera was approximately $560 \mathrm{~mm}$. The resolution of the digital camera was set as $1296 \times$ 972 pixels. The angle to the optical axis of the laser plane was set to be 26.5 degree. In this case, the vertical resolution of the laser system was approximately $0.82 \mathrm{~mm} / \mathrm{pixel}$. In addition, our experiment was made in a dark room and the camera could only receive light from the laser or light sources for an accurate quantitative evaluation.

\section{A. Experimental Calibration}

Once the experimental setup was fixed and deployed, the calibration was performed. A "dry" calibration was firstly implemented for the standard intrinsic parameters of the housing camera in air. Zhang's method [23] was used in this step. According to the two-points calibration method, we can obtain the parameters of the refractive model. We photographed a fixed checkerboard calibration plate twice both in air and water.

Following the SVP model, we estimated the real distance (ground-truth) and the virtual distance of two corner points in the checkerboard by using Zhang's method [23]. The calibrated model parameters all provided physically valid values. The refractive index, $n=1.339$, is an acceptable value for fresh water $\sim 1.333)$. And the distance from the camera origin to the housing interface, $h=63 \mathrm{~mm}$, was a reasonable value for the housing camera. All the result showed that the proposed calibration method is valid.

\section{B. Experimental Results}

1) Underwater Laser Triangulation: We firstly tested the proposed underwater laser triangulation approach. Three different methods were compared for demonstrating the validity of our approach. The first method uses the SVP model without consideration of refractive effect. The second method employs an image restoration algorithm to transform the underwater image to the air, so it can also use the SVP model. We call this method Water-to-Air (W2A) method. The third one is our method based on a proposed refractive model. 

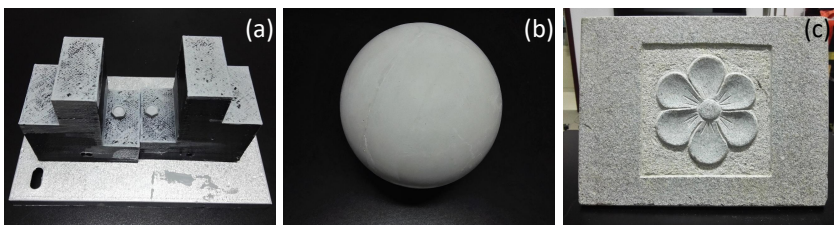

Fig. 6. Experimental samples. (a) A 3D printing sample. (b) A gypsum ball. (c) A bas-relief flagstone.
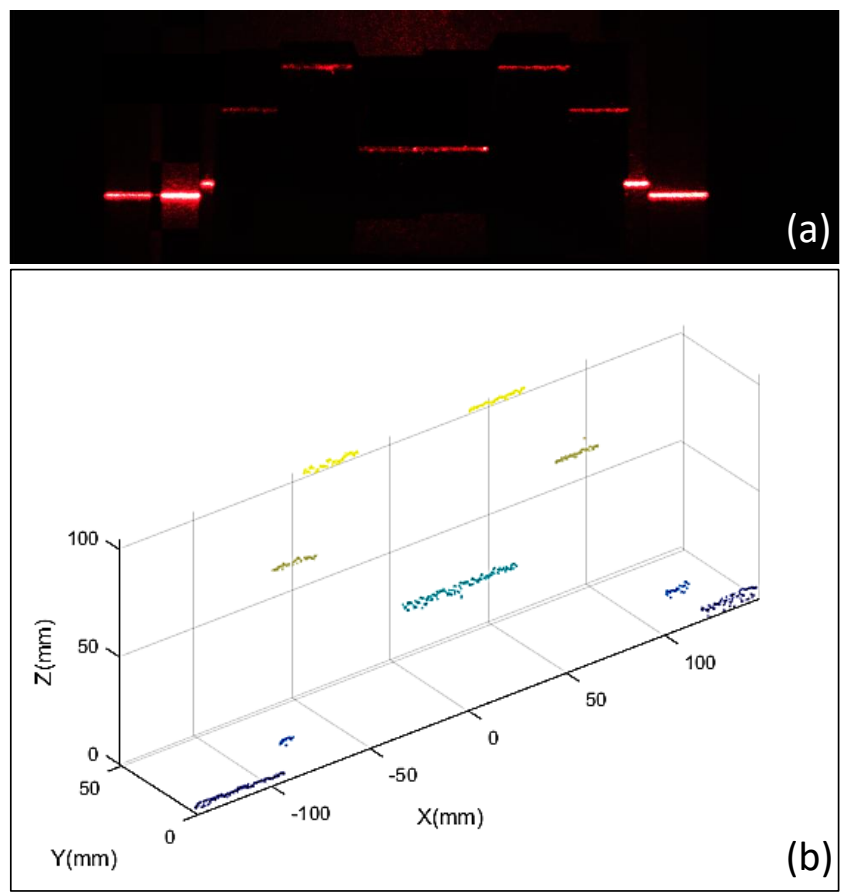

Fig. 7. Height estimation of a 3D printing sample using laser triangulation in underwater environment. (a) Laser image on the 3D printing sample. (b) The point cloud data of the 3D printing sample produced by our method.

The underwater image restoration algorithm in the second method is obtained from the proposed "virtual-real" relationship. This method is similar with Refs. [7]. As shown in Fig. 1, a scene point $\left(x_{c}, y_{c}, z_{c}\right)$ is projected into the image frame $\left(x_{u}, y_{u}\right)$ in underwater environment. Following the refractive model, a virtual point $\left(x_{v}, y_{v}, z_{v}\right)$ is related to the real point. And the real point should be projected on the image point $\left(x_{a}, y_{a}\right)$ if it was captured in air. The relationship between the air image and the underwater one is non-liner and the scale becomes larger when the distance from the image point to the origin increases [6]. The underwater image restoration algorithm can be written as:

$$
\left\{\begin{array}{l}
x_{a}=\frac{z_{v}}{z_{c}} \times x_{u}=\frac{z_{v}}{h+\left(z_{v}-h\right) \times \delta} \times x_{u} ; \\
y_{a}=\frac{z_{v}}{z_{c}} \times y_{u}=\frac{z_{v}}{h+\left(z_{v}-h\right) \times \delta} \times y_{u} .
\end{array}\right.
$$

Supposing that $h<<z_{v}$, which introduces an approximation, the relation can be simplified as:

$$
\left\{\begin{array}{l}
x_{a}=\frac{x_{u}}{\delta} \\
y_{a}=\frac{y_{u}}{\delta}
\end{array}\right.
$$

where $\delta=\sqrt{n^{2}+\left(n^{2}-1\right) \times\left(x_{u}^{2}+y_{u}^{2}\right)}$.

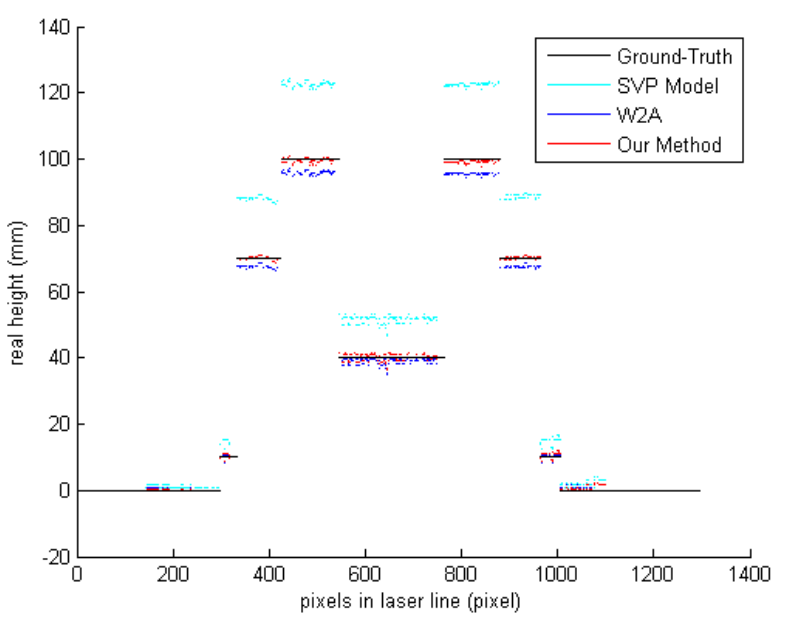

Fig. 8. Comparison of underwater laser-triangulation methods in real height.

TABLE I

ERROR ANALYSIS OF UNDERWATER LASER TRIANGULATION.

\begin{tabular}{ccc}
\hline Error units (mm) & Average error & Maximum error \\
\hline SVP Model & 12.7364 & 24.2514 \\
W2A & 2.0030 & 5.8369 \\
Our Method & $\mathbf{0 . 6 5 5 0}$ & $\mathbf{3 . 8 5 3 0}$ \\
\hline
\end{tabular}

We tested methods on a known object (Fig. 6-(a)), which was produced by a 3D printer with known height differences $(10 \mathrm{~mm}, 40 \mathrm{~mm}, 70 \mathrm{~mm}$, and $100 \mathrm{~mm})$. We used a vernier caliper to estimate the height accuracy of the 3D printing sample, and the estimated height accuracy was $\pm 0.01 \mathrm{~mm} / 10 \mathrm{~mm}$. The captured laser image of the 3D printing sample is shown in Fig. 7-(a). The comparison of different methods is shown in Fig. 8 and 9. In Fig. 8, the laser heights estimated by different methods are compared from the same view. In Fig. 9, it shows the height distributions of the three methods. The height produced by the first method is nonlinear, and the height deviation becomes larger when the pixel position is away from the center of the image plane. The second method is better than the first one. When the pixel position is close to the center, the result produced by the second method is close to the ground truth. But the height deviation becomes obvious, that the estimated height is smaller than the ground truth, when the pixel position is away from the image center. Conversely, the height estimated by our method is closer to the ground truth. And our method can accurately estimate the height difference of the tested object (Fig. 7-(b)).

Error analysis is shown in Table I. In our experimental environment, the vertical resolution of the laser system is approximately $0.82 \mathrm{~mm} /$ pixel. Compared with other methods, the average error of our method is smaller, and the accuracy is obviously improved. The average error of our method is smaller than $0.82 \mathrm{~mm} /$ pixel, which is very close to the ground truth. The deviation of our method may be caused in the calibration or laser-line extraction step, which can not be unmistakable. In conclusion, our method based on the refractive model is entirely feasible, and shows the best performance. 


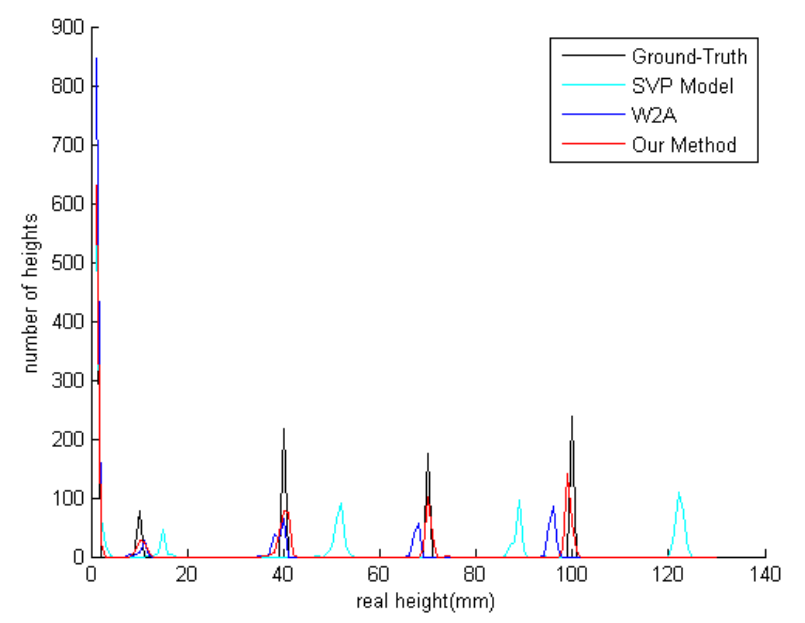

Fig. 9. Comparison of underwater laser-triangulation methods in real-height distribution

2) Underwater Photometric Stereo: We photographed a gypsum ball and a flagstone (Fig. 6-(b,c)) to validate the proposed underwater photometric stereo method. In our algorithm, some accurate points produced by underwater laser triangulation are employed to enhance the results of underwater photometric stereo.

We firstly tested on a gypsum ball. Fig. 10 shows the height of a gypsum ball produced by our method. The radius of the gypsum ball is $80 \mathrm{~mm}$. But the visible radius of the gypsum ball is less than $80 \mathrm{~mm}$, because the camera-object distance is approximately $500 \mathrm{~mm}$, which is not larger enough than the radius. The comparison of the SVP model method and our method on the gypsum ball is shown in Fig. 11. The $x$ and $y$ coordinates computed by the SVP model method are larger than the ground-truth. But the real coordinates of our method based on the refractive model is more closer to the groundtruth.

We also tested on a flagstone. Fig. 12 shows the recovered results of the flagstone. The captured image is shown in Fig. 12-(a). The vertical edge is curved because of the refraction. The real 3D mesh produced by our method is shown Fig. 12-(d). The color of the mesh denotes the height of the flagstone. Fig. 12-(b) is the recovered image from a top view. The comparison of the two figures is shown in Fig. 12-(c), that our method can correct the deformation caused by refraction.

In conclusion, experiments show that our method can provide high levels of accuracy in underwater $3 \mathrm{D}$ reconstruction.

\section{CONCLUSION}

In this paper, we put forward approaches of underwater laser triangulation and underwater photometric stereo to obtain 3D information of high accuracy in underwater environment. To remove the refractive effect, these approaches are all rebuilt based on a refractive model. In this refractive model, a "virtual-real" relationship is introduced to describe the geometric relation of the light propagation in multimedia. With the proposed geometric relation, new algorithms are respectively put forward for solving the $3 \mathrm{D}$ reconstruction problem in

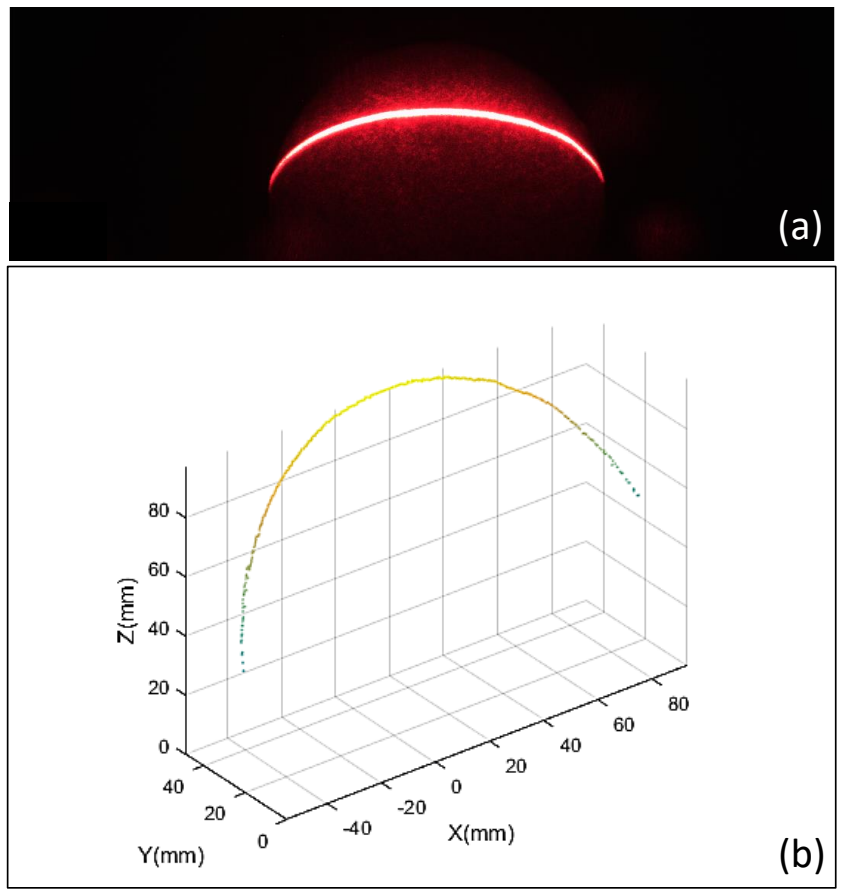

Fig. 10. Height estimation of a gypsum ball using laser triangulation in underwater environment. (a) Laser image on the gypsum ball. (b) The point cloud data of the gypsum ball estimated by our method.

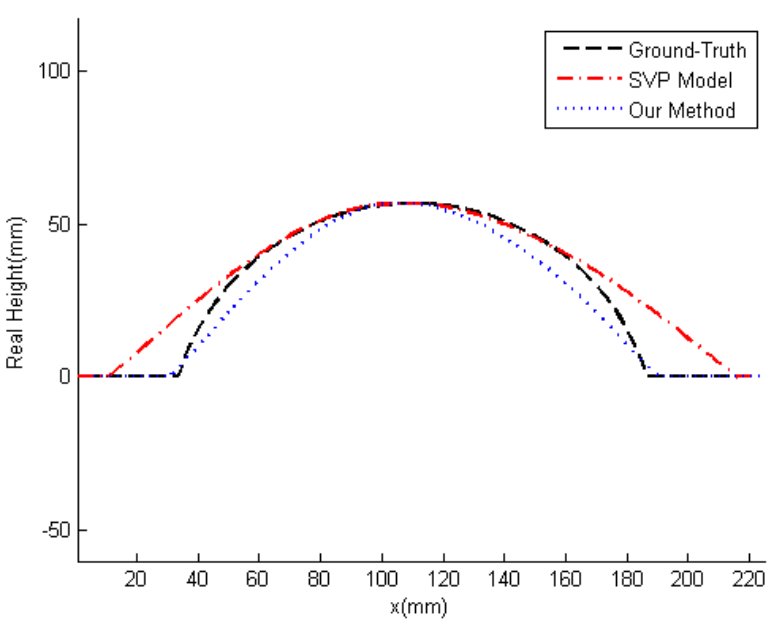

Fig. 11. Comparison of underwater photometric methods in real-height.

underwater laser triangulation and underwater photometric stereo. The proposed approaches are proven to be effective, and can also benefit other underwater applications.

In future research, we will improve our algorithm for a moving camera for larger area of reconstruction. And these extended techniques can be employed toward an underwater SLAM approach.

\section{ACKNOWLEDGMENT}

This work was supported by the International Science \& Technology Cooperation Program of China (ISTCP) (No. 

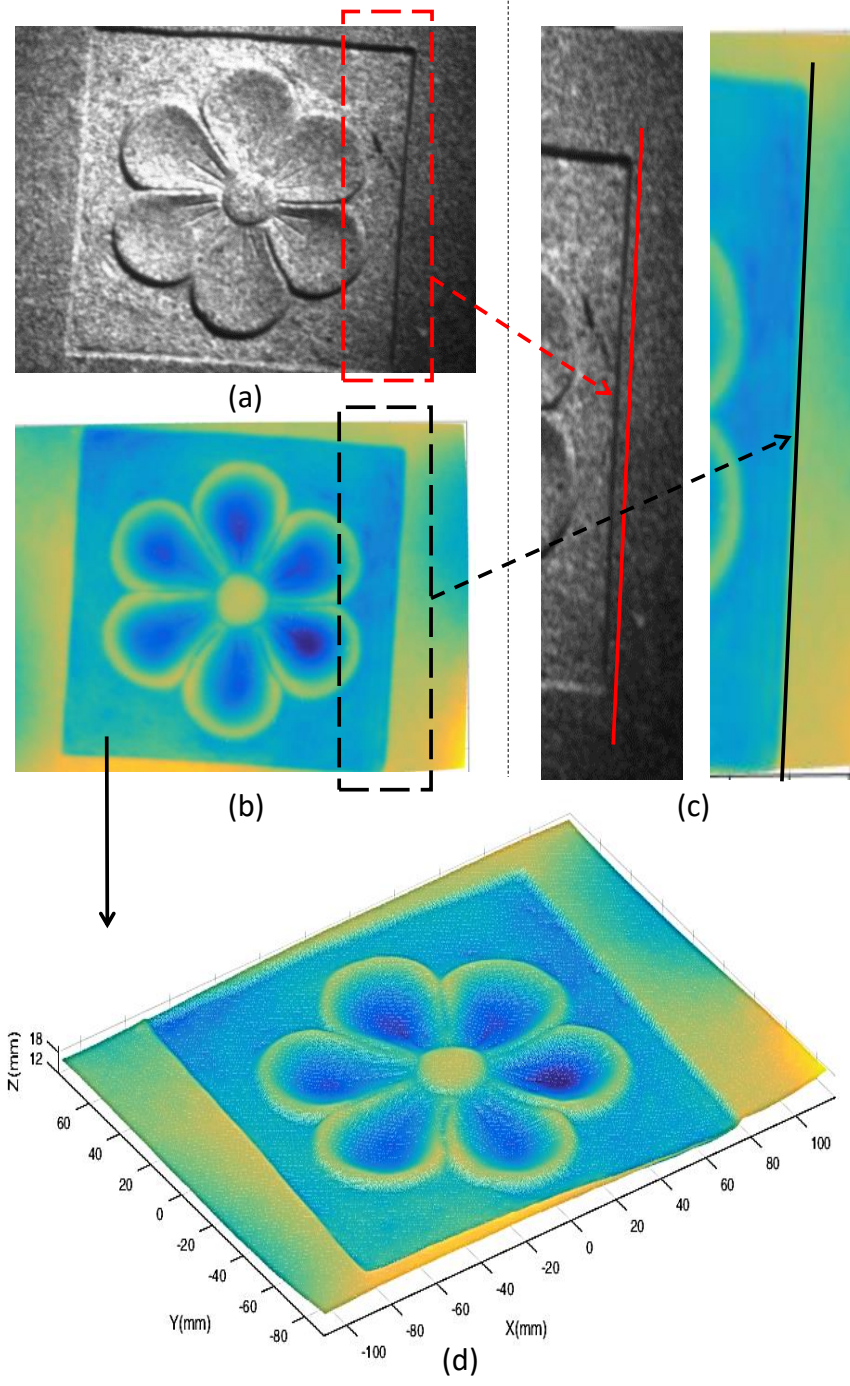

Fig. 12. The recovered figures of the flagstone by our method. (a) The captured original image. (b) The recovered mesh from a top view. (c) Comparison of this two figures. (d) The $3 \mathrm{D}$ mesh recovered by our method.

2014DFA10410) and National Natural Science Foundation of China (NSFC) (No.61501417).

\section{REFERENCES}

[1] G. Telem and S. Filin, "Photogrammetric modeling of underwater environments," ISPRS Journal of Photogrammetry and Remote Sensing, vol. 65, no. 5, pp. 433-444, 2010.

[2] S. Tetlow and J. Spours, "Three-dimensional measurement of underwater work sites using structured laser light," Measurement Science and Technology, vol. 10, no. 12, p. 1162, 1999.

[3] T. Treibitz, Y. Schechner, C. Kunz, and H. Singh, "Flat refractive geometry," IEEE transactions on pattern analysis and machine intelligence, vol. 34, no. 1, pp. 51-65, 2012.

[4] P. Drap, J. Seinturier, D. Scaradozzi, P. Gambogi, L. Long, and F. Gauch, "Photogrammetry for virtual exploration of underwater archeological sites," in Proceedings of the 21st international symposium, CIPA, 2007, p. 1 e6.

[5] C. Roman, G. Inglis, and J. Rutter, "Application of structured light imaging for high resolution mapping of underwater archaeological sites," in OCEANS 2010 IEEE-Sydney. IEEE, 2010, pp. 1-9.

[6] Y.-H. Kwon and J. B. Casebolt, "Effects of light refraction on the accuracy of camera calibration and reconstruction in underwater motion analysis," Sports biomechanics, vol. 5, no. 2, pp. 315-340, 2006.
[7] S. Chi, Z. Xie, and W. Chen, "A laser line auto-scanning system for underwater 3d reconstruction," Sensors, vol. 16, no. 9, p. 1534, 2016.

[8] R. J. Woodham, "Photometric method for determining surface orientation from multiple images," Optical engineering, vol. 19, no. 1, pp. 191 139-191 139, 1980.

[9] N. Kolagani, J. S. Fox, and D. R. Blidberg, "Photometric stereo using point light sources," in Robotics and Automation, 1992. Proceedings., 1992 IEEE International Conference on. IEEE, 1992, pp. 1759-1764.

[10] S. G. Narasimhan, S. K. Nayar, B. Sun, and S. J. Koppal, "Structured light in scattering media," in Computer Vision, 2005. ICCV 2005. Tenth IEEE International Conference on, vol. 1. IEEE, 2005, pp. 420-427.

[11] C. Tsiotsios, M. E. Angelopoulou, T.-K. Kim, and A. J. Davison, "Backscatter compensated photometric stereo with 3 sources," in Proceedings of the IEEE Conference on Computer Vision and Pattern Recognition, 2014, pp. 2251-2258.

[12] Z. Murez, T. Treibitz, R. Ramamoorthi, and D. Kriegman, "Photometric stereo in a scattering medium," in Proceedings of the IEEE International Conference on Computer Vision, 2015, pp. 3415-3423.

[13] J. S. Jaffe, "Performance bounds on synchronous laser line scan systems," Optics express, vol. 13, no. 3, pp. 738-748, 2005.

[14] F. R. Dalgleish, F. M. Caimi, W. B. Britton, and C. F. Andren, "Improved lls imaging performance in scattering-dominant waters," in SPIE Defense, Security, and Sensing. International Society for Optics and Photonics, 2009, pp. 73 170E-73 170E.

[15] K. D. Moore and J. S. Jaffe, "Time-evolution of high-resolution topographic measurements of the sea floor using a 3-d laser line scan mapping system," IEEE Journal of Oceanic Engineering, vol. 27, no. 3, pp. 525-545, 2002.

[16] C.-C. Wang, S.-W. Shyue, and S.-H. Cheng, "Underwater structure inspection with laser light stripes," in Underwater Technology, 2000. UT 00. Proceedings of the 2000 International Symposium on. IEEE, 2000, pp. 201-205.

[17] A. Jordt-Sedlazeck and R. Koch, "Refractive calibration of underwater cameras," in European conference on computer vision. Springer, 2012, pp. 846-859.

[18] H.-G. Maas, "New developments in multimedia photogrammetry," $O p$ tical 3D measurement techniques III, 1995.

[19] A. Sedlazeck and R. Koch, "Calibration of housing parameters for underwater stereo-camera rigs." in BMVC. Citeseer, 2011, pp. 1-11.

[20] T. Dolereit and U. F. von Lukas, "Calibration of shared flat refractive stereo systems," in International Conference Image Analysis and Recognition. Springer, 2016, pp. 433-442.

[21] A. Jordt-Sedlazeck and R. Koch, "Refractive structure-from-motion on underwater images," in Proceedings of the IEEE international Conference on Computer Vision, 2013, pp. 57-64.

[22] D. Forsyth and J. Ponce, Computer vision: a modern approach. Upper Saddle River, NJ; London: Prentice Hall, 2011.

[23] Z. Zhang, "A flexible new technique for camera calibration," IEEE Transactions on pattern analysis and machine intelligence, vol. 22, no. 11, pp. 1330-1334, 2000.

[24] H. Fan, Y. Luo, L. Qi, N. Wang, J. Dong, and H. Yu, "Robust photometric stereo in a scattering medium via low-rank matrix completion and recovery," in Human System Interactions (HSI), 2016 9th International Conference on. IEEE, 2016, pp. 323-329.

[25] A. Agrawal, R. Raskar, and R. Chellappa, "What is the range of surface reconstructions from a gradient field?" in European Conference on Computer Vision. Springer, 2006, pp. 578-591. 